\title{
Bienvenidos al pasado
}

Welcome to the old days

\author{
Claudia Bernazza \\ Doctora en Ciencias Sociales (FLACSO \\ Argentina); Docente-investigadora \\ de FLACSO Argentina; Docente de \\ la Universidad Nacional de Lanús. \\ Cuenta con una amplia experiencia \\ como funcionaria pública municipal, \\ provincial y nacional. Diputada nacional \\ (MC) por la provincia de Buenos Aires. \\ cbernazza@gmail.com
}

Fecha de recepción:

8.8.16

Fecha de aceptación:

15.9.16

\section{Resumen}

Los aspectos centrales del Plan de Modernización del Estado del actual gobierno nacional son discutidos desde una perspectiva que destaca su carácter instrumental, su parentesco con similares iniciativas emprendidas en la década de 1990 en Argentina y otros países latinoamericanos en el marco del ajuste neoliberal, y su directa vinculación con organismos multilaterales de financiamiento que auspiciaron dicho ajuste. Se destaca la metamorfosis de un conjunto de cuestiones típicamente políticas (los fines perseguidos por el Estado más allá de ciertas enunciaciones convencionales, las características del proceso de desarrollo impulsado desde el Estado, la participación de los trabajadores del sector público nacional y provincial) que se presentan como asuntos estrictamente técnicos y ajenos en consecuencia a la discusión o la negociación en función de diferentes perspectivas institucionales.

Palabras clave: modernización del estado - nueva gestión pública - neoliberalismo - privatizaciones - administración pública - Argentina.

\begin{abstract}
The central dimensions of the Modernization of the State Plan issued by the current government are discussed stressing its instrumental character and resemblance to similar initiatives implemented in the 1990's in Argentina and other Latin American countries as an integral part of Neoliberal economic adjustment, as well to its direct linkages to several multilateral financial organizations prompting for such adjustment. The metamorphosis of a number of typically political issues into merely technical ones, aliens to a public discussion or negotiation amongst
\end{abstract}


different institutional and substantive perspectives (such as the state goals beyond conventional generic propositions, the central characteristics of the development process and the state's role therein, participation of public sector workers and subnational jurisdictions).

Key words: state modernization - new public management - neoliberalism - privatizations - public administration - Argentina.

Estado se llama el más frío de todos los monstruos fríos.

F. Nieztsche (Así habló Zaratustra)

El Plan Nacional de Modernización del Estado constituye una de las mayores apuestas del presidente argentino Mauricio Macri y, como veremos, una fantástica muestra de todo lo que puede volver. La norma que aprueba el Plan, el Decreto $\mathrm{N}^{\circ} 434$ publicado el 2 de marzo de 2016, señala al Ministerio de Modernización como organismo responsable de su implementación. La creación de este ministerio fue una de las primeras medidas de la gestión entrante, a partir del Decreto N $^{\circ} 13$ del 10 de diciembre de 2015 que modifica la Ley de Ministerios $N^{\circ} 22.520$. Entre otras modificaciones, se crea un Ministerio que asume funciones anteriormente en la órbita de la Jefatura de Gabinete de Ministros. El decreto le asigna a la nueva cartera, entre otros temas, lo relativo al empleo público, la innovación de la gestión y el régimen de compras, imponiéndole "definir e implementar el Plan de Modernización de la Administración Pública Nacional, su administración central y descentralizada y ejercer funciones como autoridad de aplicación del mismo". Entre diciembre de 2015 y marzo de 2016, se produjeron corrimientos en las palabras -y por lo tanto en los conceptos- puesto que el Plan ya no versaría sobre la Modernización de la Administración Pública Nacional, sino del Estado. Su objetivo principal -según lo establece el Decreto No 434/16- es "convertir al Estado en el principal garante del bien común”. El objeto de la transformación es el Estado, asimilado a una Administración Pública puesta al servicio del ciudadano en un marco de eficiencia, eficacia y calidad en la prestación de servicios. El Plan está estructurado en cinco ejes: Plan de Tecnología y Gobierno Digital, Gestión Integral de los Recursos Humanos, Gestión por Resultados y Compromisos Públicos, Gobierno Abierto e Innovación Pública y, por último, País Digital. Términos como innovación, modernización, calidad, transparencia, gestión por resultados, son recurrentes a lo largo de todo el documento. 


\section{Modelos para armar}

Para examinar la propuesta modernizadora del actual Gobierno, debemos remontarnos a los principios y recomendaciones del New Public Management, la denominada Nueva Gerencia Pública, un enfoque surgido en Estados Unidos y Gran Bretaña durante la década de 1980 que se aplicó en la Argentina y otros países de Latinoamérica durante los años noventas y en los inicios del siglo XXI. El libro de Gaebler y Osborne, La reinvención del gobierno: la influencia del espíritu empresarial en el sector público (1994) le dio sustento teórico al cansancio de los ciudadanos frente a burocracias complejas y lentificadas y a la propuesta de los equipos que postularon reformas en todo el continente.

Este enfoque y el modelo de gestión que propone surgen en oposición a una serie de problemas y falencias del modelo anterior, el "normativo" o "burocrático", basado en la racionalización de la organización y el funcionamiento estatal y la distinción entre las actividades políticas y aquellas de naturaleza técnica. Este modelo, que se fue consolidando en las últimas décadas del siglo XIX y que alcanza su cenit en el siglo XX, reconoce que la autoridad política es la que controla formalmente al cuerpo de funcionarios permanentes, neutrales y anónimos. Estos funcionarios estables están altamente calificados para administrar las políticas decididas por el partido gobernante (Abal Medina y Cao 2012:38).

En contraposición a este modelo, que en el último cuarto del siglo anterior mostraba evidentes signos de agotamiento por su rigidez orgánica e inflexibilidad funcional, surge la Nueva Gerencia Pública, también denominada Nueva Gestión Pública, orientada hacia la transformación del modelo burocrático a partir de los valores y las técnicas del sector privado (Abal Medina y Cao 2012:40).

La Nueva Gerencia propuso la flexibilidad de estructuras y procedimientos, el aumento de la productividad de los trabajadores mediante estímulos al rendimiento y un enfoque centrado en la calidad. La gestión por resultados que se aplicó tanto a los agentes como a los organismos intentaba sustituir la observancia de normas y procedimientos característica del modelo tradicional por una pronta provisión de bienes y servicios. Al mismo tiempo, los textos del período comienzan a alertar sobre los niveles de corrupción de las agencias gubernamentales de los países latinoamericanos, lo que debía neutralizarse con cartas compromiso con el ciudadano y códigos de ética. Para dotar a los gerentes de mayor autonomía, se debían revisar los procedimientos burocráticos en relación con el uso de recursos presupuestarios, la contratación de personal y todo lo requerido para lograr los objetivos de las áreas u organismos. La profesionalización de estos agentes y su neutralidad política resguardaría a la gestión de cualquier desvío o tentación clientelar.

El Plan de Modernización del Estado de la actual gestión sigue casi al pie de la letra los postulados de la Nueva Gerencia Pública. Eficiencia, eficacia, calidad en la prestación de 
servicios, organizaciones flexibles, gestión por resultados, forman parte de los objetivos fundamentales del Plan. La Modernización es definida como "un proceso continuo en el tiempo que presenta acciones concretas y específicas que buscan mejorar el funcionamiento de las organizaciones públicas" a partir de la incorporación de tecnologías de la información, la reingeniería de procesos y la simplificación de procedimientos. Si bien estamos en presencia de una reforma de neto corte administrativo, la privatización de lo público aparece entre líneas, ya que todo la evoca e invoca: como en la década de 1990, se apuesta a incorporar valores y herramientas que se adjudican a la gestión empresarial para lograr una gestión más eficiente y transparente.

El plan presenta un objetivo general y objetivos específicos. Como objetivo general se pretende "alcanzar una Administración Pública al servicio del ciudadano en un marco de eficiencia, eficacia y calidad en la prestación de servicios”. Los objetivos específicos postulan “a) Orientar la administración al servicio de los ciudadanos, a través de una gestión transparente y con canales efectivos de comunicación, participación y control ciudadano; b) Promover y fortalecer el uso de las nuevas Tecnologías de Información y de las Comunicaciones para responder con mayor celeridad y efectividad a las demandas de la sociedad; c) Mejorar el desempeño de la gestión pública creando estructuras organizacionales simples y centradas en el servicio al ciudadano, que internamente se reflejen en la toma de decisiones cotidianas, articuladas con el planeamiento estratégico, el proceso de programación presupuestaria, la reingeniería de procesos, el monitoreo de gestión y la rendición de cuentas por resultados; d) Profesionalizar y jerarquizar a los empleados del Estado a través del reconocimiento de la carrera pública, del mérito y a partir del fortalecimiento de los sistemas de gestión de personal y de la ejecución sistemática de actividades de capacitación especificas a la función y aquellas orientadas a la creatividad e innovación; e) Trabajar con las provincias, municipios, la Ciudad Autónoma de Buenos Aires y otros poderes del Estado en el desarrollo de acuerdos a efectos de compartir estructuras y servicios que permitan contribuir a la modernización de las administraciones públicas". Respetando rigurosamente las reglas del arte, el plan se desagrega en cinco ejes que a su vez definen objetivos y acciones. Los ejes son: Plan de tecnología y gobierno digital, Gestión integral de recursos humanos, Gestión por resultados y compromisos públicos, Gobierno abierto e innovación pública, Estrategia País digital.

Todos estos términos pueden rastrearse en un sinnúmero de planes similares presentados en los países de la región a instancias de organismos internacionales de crédito durante las últimas décadas. Su implementación y los resultados obtenidos fueron analizados críticamente en numerosos trabajos teóricos (Bernazza 2011a, 2011b; Abal Medina y Cao 2012). Como en los casos anteriores, el Plan de Modernización del presidente Macri señala que el problema central a abordar son las cuestiones técnicas de la administración, postulando, por ende, un problema de razón instrumental. Llevando a cabo un plan enfocado en los procesos técnicos, administrativos y operativos de la gestión, se 
alcanza un Estado moderno, eficiente y eficaz en su objetivo de prestación de servicios al ciudadano. Un Estado innovador, flexible, con recursos humanos capacitados, estructuras simples e integradas merced a sistemas digitales deviene, según esta propuesta, en un Estado transparente, capaz de rendir cuentas claras y convincentes.

Lo que en términos más precisos todos los autores llaman reforma administrativa, aquí se convierte en la principal intervención de un Gobierno en materia de organización del Estado. Su preocupación abandona los debates ideológicos que impregnaron estas discusiones en el pasado, no como una omisión, sino como una convicción que conviene dilucidar.

\section{Política, gobierno y planificación del desarrollo}

El enfoque de la nueva gerencia ha sido refutado a lo largo de la última década por su incapacidad de calar hondo en la cuestión estatal. Al igual que el modelo racional clásico, sus preocupaciones se centran en cuestiones de índole técnica. El modelo burocrático y el gerencial comparten una debilidad estructural señalada en numerosas oportunidades: están desprovistos de una dimensión fundamental de todo Estado y acaso de toda organización social: la dimensión política.

El actual gobierno argentino también presenta un aparato administrador sin dimensión política. Se ofrece a la ciudadanía la ilusión de una maquinaria aceitada y perfecta, garantía de eficiencia y eticidad, negando así la complejidad de un cuerpo estatal o, lo que resulta más preocupante, negando a la política la responsabilidad de crearlo y conducirlo.

La escuela weberiana, aunque separa la racionalidad técnica de la política, pone la primera al servicio de la segunda. Como señala Cao, citando a Hughes: "Una administración bajo el control formal del liderazgo político" (Abal Medina y Cao 2012:38). La dimensión política está separada y en muchos casos resulta neutralizada, pero ejerce -o intenta ejercer- "desde afuera" y "desde arriba" el control del aparato burocrático. En esta materia, la nueva gerencia pública avanza peligrosamente hacia un "gobierno de la técnica".

En el período de posguerra y muy especialmente en la década de 1960, numerosos autores, reunidos alrededor de la intensa actividad desplegada por la Comisión Económica para América Latina y el Caribe (CEPAL) de la Organización de las Naciones Unidas, y otros espacios de reflexión y gestión de lo público, construyeron visiones que permiten pensar el Estado como una amalgama compleja entre estas dos dimensiones, la técnica y la política. Carlos Matus, un referente surgido al calor de esos debates, avanzó sobre los conceptos de gobierno y planificación definiendo un campo social surcado por conflictos. Este conflicto constituye el factum insoslayable de todo gobierno. De ahí que, según el autor, la planificación no se diseña sobre un lecho de rosas comandado por el cálculo 
científico-técnico, sino que se desarrolla en un medio resistente que se opone a nuestra voluntad. En efecto, el gobierno se ejerce sobre sujetos con distintas visiones, objetivos, recursos y poder (Matus 1987), quienes hacen su propio cálculo acerca del futuro y, a veces, tienen iguales o mayores posibilidades que nosotros de conducir el proceso social. De acuerdo con Matus, la planificación sería una cuestión técnica si sólo se refiriera a la relación de las personas con las cosas. Sin embargo, tanto el gobierno como la planificación refieren a las relaciones entre personas, las que tienen diferentes predisposiciones y comportamientos. Con esta premisa, Matus nos zambulle en las aguas profundas de la política. Allí se dirimen las relaciones de fuerza de un cuerpo social y, por lo tanto, las decisiones que configurarán un Estado.

Reducir la acción estatal a la utilización de tecnologías de administración adecuadas es la negación de las relaciones de poder que allí se despliegan. En el Plan de Modernización, el denominado Eje País Digital, postula "la elaboración de un nuevo modelo de colaboración entre las diferentes administraciones que funcione como generador de difusión y transferencia de buenas prácticas vigentes, de manera de extender el conocimiento y las experiencias exitosas en todo el territorio nacional". Este modelo parece ser un desafio de naturaleza técnica, por lo que no resulta necesario pensar en la convocatoria a mesas de concertación o cualquier otro sistema de conversación que dirima la correlación de fuerzas. Se propone "la interoperatividad e integración como intercambio de información, todo ello orientado al desarrollo de prácticas de modernización, servicios digitales, de sistemas de gestión, tecnologías de la modernización". En esta propuesta, el perro se muerde la cola: se trata de modernizar para modernizar. Silenciosa y progresivamente, se introducen valores neoempresariales y se convierten los medios en fines, perdiéndose de vista los objetivos últimos de los programas públicos (Bernazza 2011a).

La ausencia de toda alusión al Proyecto de Gobierno que da direccionalidad al Plan de Modernización, las escasas referencias a los convenios colectivos, los términos que se repiten una y otra vez a lo largo del documento, son indicadores de un enfoque instrumental "sobreactuado". El énfasis puesto en la transparencia, el acceso a la información, el gobierno abierto, contrastan con la absoluta opacidad respecto de la dimensión política del plan.

¿Modernizar para qué? La pregunta no respondida se convierte en concepción del mundo, de la sociedad y el Estado. Si la modernización es un fin en sí mismo, el mundo, los problemas sociales y la manera de superarlos están predefinidos por un pensamiento único ajeno a nosotros. El Estado puede incorporar instrumentos tecnológicos, garantizar el acceso a la información y la transparencia en la gestión. Pero más allá de estas políticas de transparencia, ¿qué sociedad se anhela? ¿Qué modelo de desarrollo se promueve? ¿Participamos en su definición? 
Reducir "la cuestión estatal" a problemas tecnológicos referidos a su administración es, en definitiva, rehuir al debate acerca del rol del Estado en materia económica y social. Asimismo, la reducción de la modernización a una cuestión técnica supone Estados sin historia, alejados de su pasado y su identidad más propia.

Para Garnier (2004), los instrumentos técnicos no son sólo técnicos, económicos o financieros, sino también y fundamentalmente políticos y, por esta razón, hábiles para el logro de objetivos de desarrollo que se postulan a partir de una determinada cosmovisión y de las necesidades y expectativas sociales que esa cosmovisión aspira a cubrir. El Plan de Modernización del Estado bajo análisis, salvo en su referencia al Estado como garante del bien común, evita opinar sobre un desarrollo que parece estar predefinido por un capitalismo al que no corresponde interpelar. La intención parece ser precisamente la despolitización de la política "sustituyéndola por algún tipo de decisión técnicamente óptima de asignación o utilización de los recursos, independiente de los diversos conflictos de intereses y de las distintas visiones que existen en la sociedad" (Garnier 2004:134). Sin embargo, tratándose de un sistema democrático en donde los conflictos y las distintas visiones deben ser incorporadas a la gestión, es menester -más allá de la promoción del control ciudadano y la participación mediante el acceso a la información- dar a conocer a la ciudadanía cuál es Proyecto de Gobierno al que responde un Plan de Modernización. Fomentar la participación ciudadana sin decir de qué manera se articularán las demandas con la voluntad de quien gobierna es, por lo menos, temerario, al jugar con el fuego de las expectativas ciudadanas que, tarde o temprano, esperan ser atendidas y satisfechas.

Un Proyecto, o si se prefiere, una Agenda de Gobierno, lejos de ser la expresión de un tipo de Estado interventor, centralista y totalitario, constituye una instancia de integración y consolidación de alianzas intersectoriales. En este sentido, obliga a la apertura de espacios de diálogo y concertación, al cálculo permanente de la correlación de fuerzas entre los diferentes actores sociales y estatales. Una tecnología del intercambio de información y de control ciudadano a través de sistemas digitales no resuelve estos problemas. Cuando no existe un Proyecto de Gobierno explícito o, lo que es más peligroso, cuando se postula una mera administración de lo instituido, la función estatal queda reducida a la formulación del presupuesto, el diseño burocrático del organigrama y la aplicación de reglamentos, inscriptos en un modelo económico y social que no corresponde interpelar (Bernazza 2006).

\section{El debate sobre el desarrollo}

El debate sobre el modelo de desarrollo fue parte de la escena política y social argentina hasta su abrupta interrupción con el golpe cívico militar de 1976. El perfil de nuestra economía regresó como debate acalorado a partir del conflicto suscitado en el año 2008 
por la Resolución 125 del Ministerio de Economía, conocido como el conflicto del campo. A raíz de la implementación de dicha Resolución -que aplicaba retenciones móviles a la soja, el girasol, el trigo y el maíz- se desencadenó un conflicto que duró 129 días y que instauró un amplio debate social, político y mediático sobre el modelo de desarrollo que se pretendía para la Argentina.

Estos debates encuentran su antecedente más inmediato en los que se iniciaron a partir de la aspiración de Estados Unidos de conducir los procesos de desarrollo en toda América. En 1947, el presidente Harry Truman se refirió ante el Congreso a la principal misión de los Estados Unidos como potencia mundial: brindar ayuda a los "pueblos libres" para que éstos puedan desarrollarse. En los países latinoamericanos, esta colaboración se reconoció con la firma de la Carta de Punta del Este en 1961, la que dio origen a la Alianza para el Progreso en el contexto de la Guerra Fría y del "peligro" que representaba la revolución cubana. Allí se acordaron reformas estructurales y planes de desarrollo que incluían reformas agrarias, fiscales y educativas, elaborados por equipos técnicos que surgieron y actuaron con el respaldo de la CEPAL. Estas propuestas fueron interpeladas desde su génesis: Ernesto "Che" Guevara, representante de Cuba en la reunión de Punta del Este, se refirió a la dicotomía entre la técnica y la política para pensar el progreso al expresar que "no puede haber técnicos que hablen de técnica cuando está de por medio el destino de los pueblos". En 1969, Fernando Henrique Cardoso y Enzo Faletto publican Dependencia y desarrollo en América Latina. Ensayo de interpretación sociológica. Allí, los autores intentan explicar las razones por las cuales a pesar del optimismo del período y de las condiciones económicas favorables, América Latina no logra los niveles esperados de desarrollo (Cardoso y Faletto 1969). Los magros resultados obtenidos obedecían, según estos autores, a razones políticas antes que económicas. Se puso de manifiesto, por primera vez, la naturaleza política de los procesos de transformación económica y por lo tanto de la planificación para el desarrollo.

Estos debates se extinguieron a partir de sucesivos golpes cívico-militares que interrumpieron los procesos democráticos del continente. A partir de la desestabilización de la región, la agenda de desarrollo se limitó a ensayar estrategias para salir del ciclo de crisis recurrentes. En nuestro país, con el advenimiento de una nueva etapa democrática, los debates sobre el desarrollo regresaron, alcanzando el primer lugar en la agenda social en ocasión del conflicto del campo. Mercado interno o apertura al mercado mundial; vivir con lo nuestro o pedir prestado; concentración del capital y teoría del derrame o redistribución del ingreso. Estos y otros debates regresaron con fuerza a la Argentina en el año 2008 a partir del conflicto del gobierno nacional con las patronales agrarias.

Este debate de altísimo voltaje puso en juego las diferentes perspectivas políticas y las diferentes visiones sobre la Argentina y su desarrollo. Desde entonces, las propuestas 
respecto del funcionamiento del Estado abren, implícitamente, un debate respecto del rol que éste debe cumplir en materia económica y de inclusión social.

\section{Planificación Estratégica y modernización tecnológica}

Cuando un Plan de Modernización propone, entre las tecnologías a implementar, la planificación estratégica, está introduciendo, necesariamente, la actividad de gobierno. Un Plan de Modernización es el resultado, necesariamente, de un Proyecto o Agenda de Gobierno. Un Plan de Modernización, cuando no revela este origen, "devela" una concepción acerca del rol del Estado. Sin tener en claro los objetivos, es difícil definir las formas de la gestión estatal.Y si el objetivo es la eficiencia, ahora convertida en un fin en sí mismo, la meta a alcanzar es un Estado mínimo. Así las cosas, la "sobreactuación" tecnológica cobra sentido: la simplificación de procesos y la robotización de la gestión oculta un proyecto de reducción y privatización de la acción estatal.

La reducción de la plantilla de empleados o la supresión de organismos dedicados a promover determinadas actividades económicas, si bien pueden presentarse como resultados de una racionalidad neutra, son el producto de una concepción precisa respecto del rol a cumplir por el Estado aun cuando no se encuentren rastros acerca de este rol en los documentos de respaldo.

En materia de "gestión por resultados", debemos tener en cuenta que los resultados a los que se alude no son un producto neutro ni deseable para la totalidad de los actores implicados. Por otro lado, aun cuando los resultados obtenidos fuesen deseables para la mayoría, es menester analizar su impacto en una realidad siempre compleja.

Asimismo, dado que las tecnologías informáticas avanzan a lo largo de la historia a partir de conflictos bélicos y que las comunicaciones se perfeccionan buscando atraer a los sujetos de la sociedad de consumo, su aplicación al ámbito estatal no puede realizarse sin las adecuaciones que reconozcan la naturaleza propia del Estado. Las tecnologías de la información y la comunicación, la mercadotecnia y la atención al cliente, entre otras, deben ser pensadas desde el "gobierno de lo público" si no se las quiere cumpliendo los objetivos para los que dichas tecnologías fueron diseñadas.

Por otra parte, muchas de las propuestas que nos invitan a consolidar la integración territorial a través del uso de tecnologías desconocen el sinnúmero de problemas de conectividad del país real. Las administraciones públicas de nuestro país son hijas de la historia y la geografia de este continente, y tanto a escala nacional, como provincial y municipal, son situadas. En la Puna, los líderes de las comisiones de fomento deben andar varios kilómetros a pie para alcanzar alguna cumbre que les posibilite obtener señal de teléfono en sus celulares. Llegar a un punto y a un dispositivo que les permita 
conectarse y cumplir con los requisitos que desde Nación o desde la provincia se les imponen, suele demandar varios días de marcha. En el oeste y sur del Gran Buenos Aires, el acceso a la oficina de ANSES más cercana o la tramitación de la tarjeta SUBE demanda horas que se restan a la salud, la escolaridad o al trabajo en el mercado informal, un mercado sin derecho a ausencias. La modernización de la Administración Pública debe contemplar la situación de cada uno de estos actores sociales, personas de a pie que dialogan muy dificultosamente con las tecnologías y los trámites. Inscribir a los recién nacidos, enterrar a los seres queridos, eximir al único inmueble que se posee de las tasas e impuestos o registrarlo como bien de familia, son trámites que se realizan frente a una administración esquiva en horarios y días de atención. Esta situación podría resolverse, según el enfoque de la nueva gerencia, abriendo oficinas digitales accesibles a través de dispositivos y servicios de conectividad que el mercado regula según las leyes de oferta y demanda, excluyentes por definición.

Desde nuestra perspectiva, la apuesta tecnológica de un Plan de Modernización debe evaluarse según su capacidad para incluir a las mayorías. Si no tiene en cuenta las dificultades de acceso a las ventanillas reales o virtuales, se ampliarán las opciones de una ciudadanía que ya accede a las tramitaciones estatales, sin correr ni un centímetro las fronteras de la inclusión.

\section{¿Recursos humanos o agentes de transformación del Estado?}

En materia de empleo público, la irrupción de la negociación colectiva en el escenario estatal abrió un panorama impensable en la década de 1990. El diálogo paritario con las organizaciones de los trabajadores, reproducido en forma intermitente y dispar en las provincias argentinas, le devolvió al colectivo de trabajadores el carácter de sujetos con voluntad e intereses autónomos respecto de la autoridad estatal, poniendo en jaque la definición de los trabajadores como "recursos humanos" de la administración. El diálogo y la concertación que se promovió desde el año 2004, a través del cual se revisaron no solamente las condiciones de trabajo, sino también el retraso y las inequidades surgidas de la implementación del Sistema Nacional de Profesionalización Administrativa (SINAPA), dio como resultado la revisión de dicho sistema y la firma del Convenio Colectivo General de Trabajo de la Administración Pública homologado por el Decreto 214 del año 2006 y la actualización del Convenio Colectivo Sectorial más abarcativo de la APN, antes SINAPA, hoy Sistema Nacional de Empleo Público (SINEP), aprobado por el Decreto 2098 del año 2008. El retraso en los concursos, las formas irregulares de contratación y otros problemas de la relación de empleo dieron lugar a un riquísimo debate en el seno del Consejo Federal de la Función Pública (COFEFUP), cuyas conclusiones se vuelcan en dos documentos de interés para el estudio del empleo público nacional y provincial: Principios y recomendaciones para una carrera profesional administrativa en las administraciones provinciales de la República Argentina, del año $2006,{ }^{1}$ y Principios y recomendaciones para las negociaciones colectivas entre el Estado y sus trabajadores, del año 2008. ${ }^{2}$ 
A pesar de estos antecedentes y la preocupación que se expresaba por las formas de contratación por fuera de un esquema de promoción y estabilidad de la relación laboral (Bernazza 2012), el Plan de Modernización que se postula en las postrimerías del 2015 se presenta sin ninguna referencia a estos debates, repitiendo lugares comunes del gerencialismo anglosajón. El regreso del término "recursos humanos" y la aparente neutralidad del discurso esgrimido no fue obstáculo -o fue vehículo- de la mayor cesación de contratos temporales de la historia del Estado argentino. Esta práctica de contratación, recomendada por los organismos internacionales de crédito y aplicada en nuestro país con la llegada de préstamos de ese origen, sumó un conjunto de trabajadores a la plantilla estatal bajo formatos flexibles, por fuera de los estatutos de personal, lo que redundó en una mayor capacidad de gestión al mismo tiempo que sumó problemas contractuales no previstos. Las inequidades entre los diferentes formatos de contratación, así como la ausencia de un horizonte de progreso, fueron en desmedro de la consolidación de equipos de trabajo comprometidos con las áreas donde se desempeñaban. A pesar de estos problemas, los sucesivos cambios de gobierno operados durante esta etapa democrática no utilizaron la cláusula anual propia de estas formas de contratación, renovando automáticamente los contratos temporales de un año al otro.

Desconociendo esta cortesía propia de un funcionariado que reconocía la calidad de trabajador público a quienes se desempeñaban bajo diferentes formas de la locación de servicios (facturando mensualmente en condición de monotributistas, las más de las veces), el Gobierno que asumió a fines de 2015 aplicó estrictamente la rescisión de contratos en forma masiva, generando un sinnúmero de situaciones que están dirimiéndose, actualmente, en la justicia laboral. El proceso se llevó a cabo sin ningún diagnóstico previo, a partir de la suposición generalizada de que todo trabajador público era el sostén de un espacio político de la gestión anterior. Con palabras pocos felices, el Ministro de Economía expresó que se debía prescindir de la "grasa" sobrante del Estado, invocando tanto al modelo de gestión lean facturing o producción "delgada" (ajustada) como al sujeto político definido por el primer peronismo. ${ }^{3}$

El documento del Programa de Análisis y Planificación de Dotación de la estrategia modernizadora dado a conocer por el diario Página $12,{ }^{4}$ cuyos puntos principales fueron corroborados por el diario La Nación en los días subsiguientes, ${ }^{5}$ se propone "producir una adecuación entre los organismos públicos y los aspectos cualitativos y cuantitativos del personal" teniendo en cuenta las personas que estarían en condición de jubilarse y las que podrían acogerse a un retiro voluntario, además de aquellos contratos que están siendo revisados en cumplimiento de lo establecido en el Decreto $N^{\circ}$ 254/2015. La aplicación de este decreto puso tempranamente en alerta a los gremios estatales más combativos, los que reclamaron por sus compañeros de ruta. Sin embargo, una opinión pública inclinada a sospechar del trabajo estatal acompañó la aplicación del decreto, a pesar de las crecientes denuncias de limitación de contratos de más de una década. Cabe señalar que a fines de 2015, al mismo tiempo que 
se producían desvinculaciones, se realizaban modificaciones al Presupuesto 2015 para sumar cargos jerárquicos a las estructuras de los organismos, aduciendo la dificultad de captar cuadros del sector privado por la baja competitividad de los salarios.

\section{Reflexiones finales}

El Plan de Modernización de la actual administración no necesita ser analizado. A lo largo de la primera década del siglo XXI, hemos señalado en numerosos trabajos las debilidades del enfoque gerencial. En la Argentina, autores de la talla de Oscar Oszlak, Horacio Cao, Daniel García Delgado, Mónica Iturburu, Carlos Vilas, Roberto Martínez Nogueira, Carlos Acuña, Hugo Cormick, Aníbal Sotelo Maciel, reunidos en grupos de investigación y cátedras universitarias, en las convocatorias del Consejo Federal de la Función Pública o en los congresos sobre la Reforma del Estado y la Administración Pública del Centro Latinoamericano de Administración para el Desarrollo (CLAD), han alertado sobre la complejidad que supone la transformación de la cultura y las prácticas de las instituciones públicas. Son autores de diferente extracción política y disciplinar, preocupados seriamente por la gestión pública. Hemos estudiado y reflexionado con ellos, hemos participado de acalorados debates donde no siempre se coincidió ni en la naturaleza ni en el origen ni en las propuestas de salida a los problemas que aquejan a las administraciones estatales a lo largo y ancho de nuestro continente. Nadie puede dudar, sin embargo, de su contracción al estudio de lo público. Sin embargo, esta producción académica e institucional no aparece como sustento de este producto inicial de la nueva Administración. Todo lo escrito a lo largo de las últimas dos décadas por estos autores puede aplicarse, en forma directa, al análisis del plan propuesto por el gobierno argentino en los albores de su gestión, tanto para señalar sus fortalezas como sus debilidades y omisiones.

Bienvenidos al pasado.

La Plata, setiembre de 2016. 
${ }^{1}$ www.sgp.gov.ar/contenidos/cofefup/documentos/docs/Principios_y_recomendaciones.pdf ${ }^{2}$ www.sgp.gov.ar/contenidos/cofefup/documentos/docs/Negociación_colectiva.pdf

${ }^{3}$ La Nación, 13 de enero 2016.

${ }^{4}$ Matías Ferrari, "Macri de nuevo con la tijera”, Página 12, 4 de setiembre de 2016.

${ }^{5}$ Agustina López, "El gobierno hará una revisión integral de la planta de empleados”, La Nación, 7 de setiembre de 2016 . 


\section{Referencias bibliográficas}

Abal Medina, Juan Manuel y Horacio Cao (2012) Manual de la nueva administración pública Argentina, Buenos Aires: Ariel.

Bernazza, Claudia A. (2006) La planificación gubernamental en Argentina. Experiencias del período 1974-2000 como puntos de partida hacia un nuevo paradigma. Tesis de Doctorado. Disponible en: http://www.claudiabernazza.com.ar/web/actividad-academica.

Bernazza, Claudia A. (2011a) "Un Estado al servicio del Proyecto Nacional”, en Revista Reseñas y Debates $N^{\circ} 70$, diciembre de 2011.

Bernazza, Claudia A. (2011b) “¿Qué Estado necesitamos? Una gestión pública para el desarrollo con inclusión”, en Daniel García Delgado y Miguel Peirano (comps.), El modelo de desarrollo con inclusión social. La estrategia de mediano plazo, Ciccus - IDISAFLACSO.

Bernazza, Claudia A. (2012) "Administración y Compromiso. Los desafíos del empleo público”, en Tiempo Argentino, 6 de julio.

Cardoso, Fernando Henrique y Enzo Faletto (1969) Dependencia y desarrollo en América Latina, Buenos Aires: Siglo XXI Editores. Disponible en: http://doctoradosociales. com.ar/wp-content/uploads/2016/04/Cardoso-y-Faletto-1970.pdf.

CONSEJO FEDERAL DE LA FUNCIÓN PÚBLICA (2006) Principios y recomendaciones para una carrera profesional administrativa en las administraciones provinciales de la República Argentina, Mendoza, 23 y 24 de noviembre de 2006. Disponible en http://www.sgp. gov.ar/contenidos/cofefup/documentos/docs/Principios_y_recomendaciones.pdf

CONSEJO FEDERAL DE LA FUNCIÓN PÚBLICA (2008) Principios y recomendaciones para las negociaciones colectivas entre el Estado y sus trabajadores, Termas de Río Hondo, 28 de agosto de 2008. Disponible en http://www.sgp.gov.ar/contenidos/cofefup/documentos/docs/Negociacion_colectiva.pdf

Gaebler, Ted y David Osborne (1994) La reinvención del gobierno: la influencia del espíritu empresarial en el sector público, Buenos Aires: Paidós.

Garnier, Leonardo (2004) "El espacio de la política en la gestión pública”, en Luiz Bresser-Pereira, Nidia Cunill Grau, Leonardo Garnier, Oscar Oszlak y Adam Przeworski (2004) Política y gestión pública, Buenos Aires: CLAD/ Fondo de Cultura Económica. 
López, Agustina (2016) “El gobierno hará una revisión integral de la planta de empleados", La Nación, 7 de setiembre de 2016. Disponible en http://www.lanacion. com.ar/1935275-el-gobierno-hara-revision-integral-planta-empleados.

Matus, Carlos (1987) Política, planificación y gobierno. Caracas: Fundación ALTADIR.

\section{Cómo citar este artículo}

Bernazza, Caludia: Bienvenidos al pasado. Revista Perspectivas de Políticas Públicas (2016) Vol. 6, N 11:23-37. 